\title{
One year on
}

\author{
The most important \\ aspect of the $B D J$ is that \\ more of each issue is \\ relevant and \\ understandable to \\ more people. If we \\ have achieved this aim \\ then the new design \\ has succeeded.
}

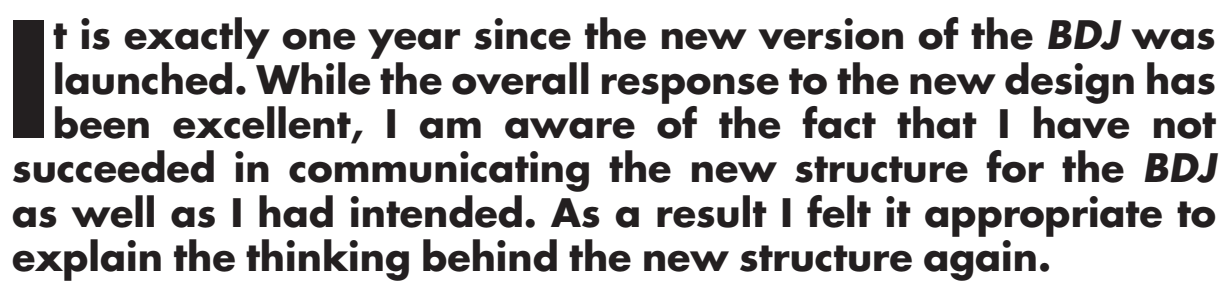

The main fundamental change to the Journal is the division into six separate sections, with each section having a different function. These six sections (in order from the front to the back of the Journal) are 'opinion', 'practice', 'summaries', 'research', 'education' and 'news and notes'.

The 'opinion' section contains the leader and any guest leaders, articles expressing a personal opinion and the 'letters to the editor'. The emphasis in the section is on the personal views of the authors on any dental subject. Most manuscripts in this section (and some letters) are subject to a fairly general refereeing process to ensure as much accuracy as possible.

The 'practice' and 'research' sections are intended for manuscripts containing material for information on topics that are very relevant to dentistry with an emphasis on clinical practice, and papers for both sections are subject to stringent peer review. However, both sections have very different functions and guidelines. The 'research' section is intended to be a forum for researchers and scientists to communicate their findings to each other. The 'practice' section is intended for all those who practice dentistry (not just general dental practitioners) thus enabling authors to communicate the application of both research findings as well as clinical techniques based on sound principles. Political, management and administrative subjects would also fit into this section.

Between the 'practice' and 'research' section you will find 'summaries'. This section contains summaries of abstracts from both the literature generally as well as the research papers in the same issue of the $B D J$. This unique arrangement provides a quick summary and comment on each research paper to help readers obtain the basic information quickly, an important aspect today as the amount of material we receive continues to increase.

The 'education' section contains any form of paper that focuses on dental education, thus enabling opinion, practice or research papers to be published here. This section does not automatically appear in every issue as we do not receive enough material to enable us to do so, but does mean that papers on dental education no longer have to wait until October to be published as used to happen in the days of the 'Educational Issue'.

Finally the 'news and notes' section contains just that - news and notes on a variety of topics that have traditionally been in the Journal since its inception in 1880.

The area of most confusion (from an author's viewpoint) seems to be the fact that 'research' and 'practice' papers do not have to be aimed at general practitioners in order to be accepted. Admittedly, this appeared to be the case in the past, a fact that is understandable in view of the fact that the $B D J$ readership contains far more GDPs than other dental groups. However, the new design has tried to create a journal that should still appeal to GDPs because of the increase in practice papers without restricting itself to this group. In the 'research' section the scientists now have the opportunity to submit high quality science aimed at informing researchers in other disciplines as well as papers aimed at GDPs, increasing the value of this section to all.

The most important aspect of the BDJ (I hope) is that more of each issue is relevant and understandable to more people. If we have achieved this aim then the new design has succeeded. 